\title{
Editorial: Software-Defined and Virtualized Future Wireless Networks
}

\author{
Yong Li • Athanasios V. Vasilakos
}

Published online: 3 February 2015

(C) Springer Science+Business Media New York 2015

\section{Editorial:}

While the past few decades have witnessed the rapid growth in mobile and wireless networks, numerous problems and challenges become increasingly serious, such as heterogeneous wireless networks, spectrum scarcity, smooth evolving and fast deployment, technologies innovations, QoS and QoE support, etc. Traditional mobile and wireless network technologies can hardly overcome these challenges.

Software defined network, an innovative paradigm, has become one of the hottest topics in wired and wireless networking. It advocates separating the control plane and data plane, and abstracting the control functions of the network into a logically centralized control plane. However, key technologies in software defined wireless networks (SDWN) are still open, including the end-to-end architecture, design and implementation of wireless technologies, software defining strategies, etc. At the same time, cloud computing and network virtualization are considered as a promising way that benefits future wireless networks, such as using the cloud computing pool providing powerful computing capability to implement the baseband data processing of wireless communication. However, how to design, customize, and utilize the

\section{Y. $\operatorname{Li}(\square)$}

Huazhong University of Science and Technology, Wuhan, China e-mail: liyong07@tsinghua.edu.cn

\section{A. V. Vasilakos}

University of Western Macedonia, Athens, Greece

A. V. Vasilakos

National Technical University of Athens (NTUA), Athens, Greece virtualization and cloud computing technologies targeted for the wireless networks are still open problems.

The special issue focuses on the architecture and key technologies of software-defined and virtualized future mobile and wireless networks. We have received $40+$ submissions, and finally accepted 8 papers to publish in this SI. At the same time, we have also selected 4 high quality best papers from International Conference on Software-Defined and Virtualized Future Wireless Networks, which held in Rome, Italy, October 27, 2014. All these papers together investigated and discussed the following five major topics: network architectures for SDWN and virtualization, SDN wireless backhaul and IP network, resource allocations for SDWN, applications of SDWN, and corresponding security and charging problems.

For the network architectures of SDWN and virtualization, the first article, "Software-Defined and Virtualized Future Mobile and Wireless Networks: A Survey", co-authored by Mao Yang, Yong Li, Depeng Jin, Lieguang Zeng, Xin Wu and Athanasios V. Vasilakos, discusses the challenges and expectations of current wireless networks, and analyzed the opportunities provided by the SDWN and wireless network virtualization (WNV). Then, this paper focuses on SDWN and WNV by presenting the main ideas, advantages, ongoing researches and key technologies, and open issues respectively. Furthermore, this paper interprets these two technologies highly complement each other, and investigates efficient joint design between them. This work confirms that SDWN and WNV may efficiently address the crucial challenges and significantly benefit the future mobile and wireless network. The second article, "Multi-Perspective Virtualization and Software-Defined Infrastructure Framework for Wireless 
Access Networks", co-authored by Heming Wen, Prabhat Kumar Tiwary and Tho Le-Ngoc, presents Aurora, a virtualization framework and testbed platform for supporting multiple types of virtualization techniques and architectures specifically applied to wireless technologies. Aurora is designed to fulfill multiple roles as a powerful tool to combine multiple wireless virtualization technologies, a softwaredefined research platform for developing new virtualization architectures and a service-oriented wireless infrastructure manager.

For the topic of SDN wireless backhaul and IP network, article, "Integrating WMN based mobile backhaul with SDN control", co-authored by Kari Seppanen, Tapio Suihko and Jorma Kilpi, provides solution of suitable backhaul connections for future broadband mobile networks by SDN-based wireless mesh networks (WMNs). This solution benefits the centralized controlling of SDN, and it is best suited in cases where base stations are installed in locations without optical fiber connection to transport network, e.g., small-cell deployment to hot spots in dense urban areas. On the other hand, article, "Design and Implementation of A Software-defined Mobility Architecture for IP Networks", co-authored by You Wang, Jun Bi and Keyao Zhang, applys the Software Defined Networking (SDN) to mobile and wireless networking to make them adapt to the rapid development and popularity of the mobile Internet. It focuses on extending SDN paradigm to mobility handling in the mobile Internet by designing and implementing and deployment of a software-defined IP mobility architecture.

For the topic of resource allocations for SDWN, the article, "Converged management in heterogeneous wireless networks based on resource virtualization" co-authored by Luhan Wang, Zhaoming Lu, Xiangming Wen and Wanqing Guan, focuses on the resource management in Dense heterogeneous networks (HetNets), which are supposed to be one of the most inevitable modalities of future radio access networks (RAN). This paper proposes a converged management architecture for HetNets (CMA) based on resource virtualization, which whittles traditional HetNets management down to the procedure of virtual resources allocation by demonstrating some use cases. The article, "Traffic Aware Cross-Site Virtual Machine Migration in Future Mobile Cloud Computing", co-authored by Jiaqiang Liu, Yong Li, Depeng Jin, Li Su and Lieguang Zeng, focuses on the scenarios of mobile cloud computing, which is a new paradigm to provide Internet services to mobile users. By moving Virtual Machines(VMs) to the sites closest to their users, cross-site VM migration is promising to improve users' experience. This paper studies the problem of VM migration sequence planning problem as a Mixed Integer Linear Programming (MILP) problem considers both inter-VM communication traffic and migration traffic to optimize the usage of network resources in terms of bandwidth and computation.

For the topic of applications of SDWN, the first article, "Cognitive Internet of Things: A Unified Perspective", coauthored by Syed Zaidi, Muhammad Shakir, Muhammad Imran, Mounir Ghogho, Thanos Vasilakos], Khalid Qaraqe and Des McLernon, presents a unified perspective on the cognitive internet of things (CIoT). By unifying these distinct technologies into a CIoT architecture that provides a flexible, dynamic, scalable and robust network design road-map for a large scale IoT deployment, this paper proposes a general statistical framework and new metrices so that the design space of the CIoT can be quantitatively explored in the future. The article, "Suboptimal Beam Search Algorithm and Codebook Design for Millimeter-Wave Communications", coauthored by He Tong and Zhenyu Xiao, discusses the codebook design problem in the environment of software-defiend Millimeter-Wave (mmwave) communication, which introduces the mmwave communications and networks as a main applications of SDWN. The article, "The Characterizes of Communication Contacts Between Vehicles and Intersections for Software-Defined Vehicular Networks", co-authored by XueFeng Xiao and Xiaoyan Kui, introduce vehicular networks as the SDWN's application. In software-defined vehicular networks, a centralized controller in the network through the road-side unit will greatly promote the efficiency of the network efficiency by providing highly reliable transmission. By modeling the inter-contact time and contact duration, this paper makes way for the future study and the deployment of software-defined vehicular networks.

For the topic of security and charging problems, the article, "Secure Communication over Software-Defined Networks", co-authored by Stefan Rass, Benjamin Rainer, Matthias Vavti, Johannes Göllner, Andreas Peer and Stefan Schauer, reports on work in progress towards a practical implementation of a software defined overlay network that provides data delivery services at a freely definable and provably optimized quality of service. The article, "Towards Active Charging for Software Defined Wireless Networks", co-authored by Brian Lee, Yuansong Qiao and Niall Murray, considers how earlier approaches of SDWN to active charging can be combined with the 3GPP charging framework and recent SDN inspired cellular architectural innovations to provide an active charging infrastructure for SDN. 
The guest editors are thankful to our reviewers for their effort in reviewing the manuscripts. We also thank the Editin-Chief, Dr. Imrich Chlamtac for his supportive guidance during the entire process.

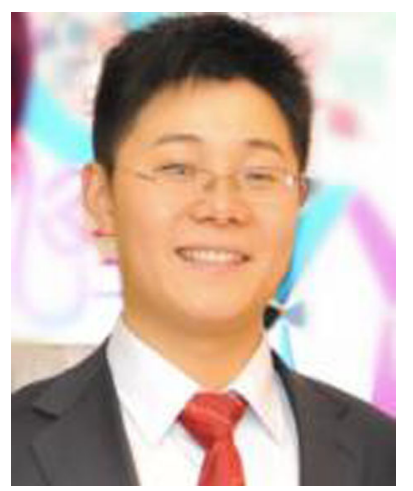

Dr. Yong Li received his B.S. degree in electronics and information engineering from Huazhong University of Science and Technology, Wuhan, China, in 2007 and his Ph.D. degree in electronic engineering from Tsinghua University, Beijing, China, in 2012. In July to August of 2012 and 2013, he was a visit- ing research associate with Telekom Innovation Laborato- ries and the Hong Kong University of Science and Technology, respectively. From December 2013 to March 2014, he was a visiting scientist with the University of Miami, Coral Gables, Florida. $\mathrm{He}$ is currently a faculty mem- ber of the Department of Electronic Engineering, Tsinghua University. His research interests are in the areas of net- working and communications, including mobile oppor- tunistic networks, device-to-device communication, software-defined networks, network virtualization, and future Internet. He has served as a Technical Program Com- mittee (TPC) Chair for the WWW workshop of Simplex 2013 and on the TPCs of several international workshops and conferences. He is a Guest Editor of ACM/Springer Mobile Networks and Applications, Special Issue on Soft- ware-Defined and Virtualized Future Wireless Networks. He is currently an Associate Editor of EURASIP Journal on Wireless Communications and Networking. He was a recipient of the Outstanding Postdoctoral Researcher, Outstand- ing Ph.D. Graduates, and Outstanding Doctoral Thesis awards from Tsinghua University. His research is granted by the Young
Scientist Fund of the Natural Science Foundation of China, the Postdoctoral Special Fund of China, and industry companies Hitachi, ZET, and others.

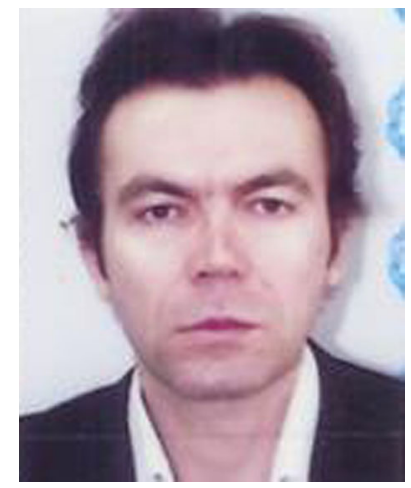

Dr. Athanasios V. Vasilakos is currently Professor at the University of Western Macedonia, Greece and Visiting Professor National Technical University of Athens (NTUA), Athens, Greece. He has au- thored or coauthored over 200 technical papers in major international journals and conferences. He is author/coauthor of five books and 20 book chapters in the areas of communications. Prof. Vasilakos has served as General Chair, Technical Program Committee Chair for many international conferences. He served or is serving as an Edi-tor or/and Guest Editor for many technical journals, such as the IEEE TRANSACTIONS ON NETWORK AND SERVICES MANAGEMENT, IEEE TRAN SACTIONS ON SYSTEMS, MAN, AND CYBERNETICSPART B:CYBERNETICS, the IEEE TRANSACTIONS ON INFORMA- TION TECHNOLOGY IN BIOMEDICINE, ACM TRANSACTIONS ON AUTONOMOUS AND ADAPTIVE SYSTEMS, the IEEE JSAC special issues of May 2009, Jan 2011, March 2011, the IEEE Communications Magazine, ACM/ Springer Wireless Networks(WINET), ACM/Springer Mo- bile Networks and Applications(MONET). He is founding Editor-inChief of the International Journal of Adaptive and Autonomous Communications Systems (IJAACS, http://www.inderscience.com/ijaacs) and the International Journal of Arts andTechnology (IJART,http:// www.inderscience.com/ijart). He is General Chair of the Council of Computing of the European Alliances for Innovation. 